\title{
Correction of Shallow Water Electromagnetic Data for Noise Induced by Instrument Motion
}

\author{
Pamela Lezaeta, Alan D. Chave and Rob L. Evans \\ Woods Hole Oceanographic Institution, Woods Hole, MA 02543, USA
}

(December 13, 2004)

Short title: Correction of Electromagnetic Motion Noise

\begin{abstract}
An unexpected noise source was found in magnetic and sometimes electric field data recorded on the bottom of lakes in the Archean Slave craton (NW Canada) during warm seasons. The noise is due to instrument motion and in some instances direct induction by wind-driven surface gravity waves when the lakes are not ice covered. The noise can be reduced or eliminated by pre-filtering the data with an adaptive correlation noise cancelling filter using instrument tilt records, prior to estimation of magnetotelluric (MT) response functions. Similar effects are to be expected in other shallow water environments, and the adaptive correlation canceller is a suitable method to pre-process MT data to reduce motional noise in the magnetic field. This underscores the importance of ancillary tilt measurements in shallow water MT surveys. In coastal or lake bottom surveys, special efforts to reduce hydrodynamic effects on the instrument should also be pursued.
\end{abstract}

\section{INTRODUCTION}

During the years 1998-2000, two magnetotelluric (MT) surveys were conducted on the Archean Slave craton in eighteen lakes over nineteen deployments using continental shelf electromagnetic instrumentation designed for use at depths lower than 
$1000 \mathrm{~m}$ (Pettit et al., 1994). The instruments were deployed from float airplanes during the ice-free summer months and retrieved in the subsequent summer. The first sequence of lakes was surveyed for eleven months, at which point the instruments were retrieved and transferred to a second series of lakes for another year. The measurements were aimed at determining the regional-scale three-dimensional (3-D) electrical structure of the craton (Jones et al., 2003). During the first month of each survey, the data were recorded at a high sample rate $(1.75 \mathrm{~s})$ for imaging crustal features. The instruments then switched automatically to a lower sample rate $(28 \mathrm{~s})$ for the remainder of the deployment to obtain long period MT data, permitting deep penetration into the lithospheric mantle and below. Each instrument was programmed to record the time variations of the two orthogonal horizontal electric fields across a $3 \mathrm{~m}$ span using $\mathrm{Ag}-\mathrm{AgCl}$ electrodes, and the three-component magnetic field variations were measured using a suspended magnet sensor (Filloux, 1987). The horizontal plane tilt variations of the cylindrically shaped magnetometer pressure case were also measured with microradian resolution. Figure 1 shows a picture of an instrument being deployed. Instrument installation depths ranged from 7 to $50 \mathrm{~m}$, with 15-20 m being a typical value. Time series of tilt and magnetic field were stable in wintertime except for normal geomagnetic variations, while they displayed strongly disturbed intervals from late spring through summer and into early fall. The disturbance was particularly noticeable in the high sample rate data, where short period tilt variability correlated strongly with the magnetic field (and sometimes the electric field) fluctuations. Such noise was somewhat unexpected, as the lakes were expected to be calm with weak water currents in comparison to the continental shelves.

This paper focuses on the analysis of nonstationary instrument motion that affected the magnetic (and sometimes the electric) field data, and on the development of a time-domain mitigation technique for the correlated noise using an adaptive correlation cancellation filter prior to estimation of frequency-domain MT response functions. We also propose a physical explanation for the cause of the instrument 
motion and suggest a possible means to minimize the problem.

\section{DATA SET}

Figure 2 shows the location of the lake bottom sites. In the first survey, from $\mathrm{Au}-$ gust 1998 to August 1999, ten sites were recorded, and nine more sites were occupied in the second year, from September 1999 to August 2000.

The electric and magnetic channels were edited to correct for obvious instrument effects (such as infrequent data spikes and regular magnetic field calibration pulses) and high pass filtered to remove long term drift using a fourth order Butterworth filter having a 24,000 s cutoff period run forward and backward across the data to eliminate possible phase shifts. Due to inevitable and uncontrollable electrode drift, the two electric channels occasionally were out of range even with gain ranging applied, producing some data gaps in one or both components. The magnetic time series were continuous. Figure 3 shows examples of magnetic and electric time variations from Newbigging Lake (site newBig in Fig. 2) for a $10 \mathrm{~h}$ window at the high $(1.75 \mathrm{~s}) \mathrm{sam}-$ ple rate. During the first six hours, the data have a higher frequency character than during subsequent hours, particularly for the magnetic field. The magnetic field and tilt variations approximately covary during the first six hours of the window (Fig.4). Many sites displayed similar correlations in the high sample rate time series during the summer months.

\section{TILT NOISE CORRELATION WITH FIELD COMPONENTS}

The corresponding components of tilt and magnetic field vary coherently when the high frequency disturbance is present. This disturbance was generally at least twice as large for the component (Y) across the magnetometer cylinder compared to the along-cylinder $(\mathrm{X})$ component. Although not truly periodic, the variations have a dominant period of about $4.5 \mathrm{sec}$ which becomes clear for short time windows 
(Fig.5). The electric field (E) is usually not correlated with the disturbed tilt except in extreme cases. Figure 6 shows an example for the Y component from Newbigging Lake, while the $E_{x}$ field seems unaffected by the tilt motion (Fig.6; top). The electric field perturbation when present lasted from a few minutes to as long as several hours, also with a period of $\sim 4.5 \mathrm{~s}$.

It is well known that mechanical motion of a magnetometer in Earth's magnetic field will generate an apparent magnetic field (e.g., Bastani and Pedersen, 1997), and hence correlated noise, but this should not affect the electric field unless $\mathbf{v x B}$ is comparable to the ambient field (Eq.6) and there is local induction in the surrounding medium, as occurs in the highly conductive ocean for a variety of water disturbances. Further discussion is presented in a later section.

Power spectra for the magnetic field and tilt computed from perturbed and quiet time segments (Fig.7) show that tilt motion systematically affected the magnetic field at periods below $1000 \mathrm{~s}$, with peak amplitudes centered below $10 \mathrm{~s}$ (Fig.7), consistent with the time series plots. During disturbed times, the high frequency magnetic field noise floor rises by up to four decades in power, consistent with the change for tilt. The tilt motion also systematically affected the vertical magnetic field component by around one to two decades in power, while it was not significant in the power spectrum of the electric field components for the majority of the sites.

\section{NOISE REMOVAL WITH AN ADAPTIVE CORRELATION CANCELLATION FILTER}

The tilt-induced noise is highly variable in time, and hence removal using frequency-domain transfer function methods implicitly dependent on the stationarity assumption are of little use. Time-domain data adaptive filtering methods have been developed that are applicable to non-stationary situations, of which the most useful in 
the present context is the adaptive correlation canceller (Widrow and Stearns, 1994). The adaptive correlation canceller incorporates time-variable filter coefficients which change in an optimal sense to remove unknown interference contained in a primary signal, using a noise reference in which the primary signal is weak or nonexistent (Fig.8). In the present case, the primary, contaminated signal is a component of the magnetic (or sometimes, electric) field and the reference (noise) signals are the two horizontal components of tilt. Each adaptive filter is a linear transversal (finite impulse response) filter whose coefficients are updated at each time step using the least mean square (LMS) algorithm.

Let the primary signal be given by:

$$
S=S_{o}+n_{o}
$$

where $n_{o}$ represents the noise in $\mathrm{S}$ that is correlated in an unknown manner with the corrupted signals or reference noise $\operatorname{Re} f 1$ and $\operatorname{Ref} 2$ (i.e., the two tilt components). The signal free of such noise is thus given by $S_{o}$.

At the discrete time $\mathrm{m}$, let the predicted noise $\mathbf{y}(\mathrm{m})$ be the sum of the outputs of the adaptive filters, let the adaptive filter coefficients be $W 1(\mathrm{~m})$ and $W 2(\mathrm{~m})$, and let the reference signals be $\operatorname{Re} f 1(\mathrm{~m})$ and $\operatorname{Re} f 2(\mathrm{~m})$ (Fig.8). Then, the output of the adaptive filter at time $\mathrm{m}$ is

$$
\mathbf{y}(\mathrm{m})=\mathrm{W} 1(\mathrm{~m}) \cdot \operatorname{Ref} 1(\mathrm{~m})+\mathrm{W} 2(\mathrm{~m}) \cdot \operatorname{Ref} 2(\mathrm{~m})=\mathbf{W}(\mathrm{m}) \cdot \boldsymbol{\operatorname { R e f }}(\mathrm{m})
$$

Using the LMS algorithm, at time step $\mathrm{m}+1$, the filter ( $\mathbf{W}$ in equation 2 ) is given by:

$$
\mathbf{W}(\mathrm{m}+1)=\mathbf{W}(\mathrm{m})+\frac{\mu \cdot \operatorname{err}(\mathrm{m}) \cdot \boldsymbol{\operatorname { R e f }}(\mathrm{m})^{\mathrm{t}}}{\alpha+\sum_{\mathrm{i}=1}^{\mathrm{N}} \operatorname{Ref}(\mathrm{m}-\mathrm{i})^{2}}
$$

with $\mathrm{t}$ representing the transpose of $\mathbf{R e f}$ and $\mathrm{N}$ the filter length. The coefficient $\mu$ (equation 3) is the adaption step size of the time domain filter and $\alpha$ is a damping factor, both of which are found iteratively by minimizing the sum of squared differences between the input data and the predicted noise $\left(\operatorname{err}^{2}(\mathrm{~m})\right.$; equation 4) over the 
time space of input data $\mathrm{m}(\mathrm{m}=1, . ., \mathrm{Nd}$; number of data). The output signal at the discrete time $\mathrm{m}$ is the difference between the primary input signal $\mathbf{X}_{-}$in delayed by a given time $(\mathrm{N} / 2)$ and the filter output signal $\mathbf{y}(\mathrm{m})$ :

$$
\operatorname{err}(\mathrm{m})=\mathbf{X}_{-} \text {in }(\mathrm{m}-\mathrm{N} / 2)-\mathbf{y}(\mathrm{m})
$$

where $\mathrm{N} / 2$ is a delay time introduced to "grow" the coefficients toward the center of the filter ( $\mathbf{W}$; equation 3), so that if timing between the input primary signal $\mathbf{X}_{\text {_in }}(\mathrm{m})$ and the reference noise (Ref) is not aligned, the correlator $\mathbf{W}$ will be able to find the correct value (within the interval $\pm \mathrm{N} / 2$ of the filter length $\mathrm{N}$ ). The starting set of adaptive filters $\mathbf{W}(\mathrm{m})$ (i.e., for $\mathrm{m}=1, . ., \mathrm{N}$ ) are initialized to zero at a first step (each step is over the time space Nd of input data), so that for further steps, W will start from the previous result, computing the following $\mathbf{W}(\mathrm{m})$ as in equation 3 to get the output $\operatorname{err}(\mathrm{m})$ (equation 4) from the sum of predicted noise (eq.2) over the filter length $(\mathrm{N})$ :

$$
\mathbf{y}(\mathrm{m})=\sum_{\mathrm{i}=1}^{\mathrm{N}}(\mathbf{W}(\mathrm{m}-\mathrm{i}) \boldsymbol{\operatorname { R e f }}(\mathrm{m}-\mathrm{i}))
$$

When the variance of $\operatorname{err}\left(\sum_{m=1}^{N d} \frac{\operatorname{err}(\mathrm{m})^{2}}{N d-1}\right)$ has changed minimally from the previous step, then the final $\operatorname{err}(\mathrm{m})$ is considered to be the clean output $\mathbf{X}_{-}$out(m) (ideally $S_{o}$; equation 1) at the discrete time m. Because of the large dynamic range of the time series, better performance is typically achieved by first prewhitening each time series using a robust autoregressive (AR) filter (Chave and Thomson, 2004).

The application of the noise canceller to the Slave lakes data gave, in general, satisfactory results. Figure 9 shows segments of noise-cancelled magnetic field signals along with the original (prewhitened) disturbed time series from Lake Tete d'Ours from the first year survey. Tilt amplitudes (also prewhitened with the AR filter) for this site varied by up to 200 microradians in the time interval selected (from 13th to 15th of August). The electric field within this interval was not affected by tilt, suggesting purely mechanical (non-inductive) noise on the magnetic channels. 
The noise canceller did not always fully succeed on magnetic time series having extreme disturbance levels, which usually occurred when the electric field channels were also affected by tilt. Figure 10 shows such magnetic and electric time series from Aylmer Lake. This site was the shallowest deployment of the survey at $6 \mathrm{~m}$, and both tilt components have similar amplitudes of up to 250 microradians. The adaptive correlation filter achieved only a partial reduction in the peak time series amplitude, thus reflecting limited cancellation of the tilt-induced noise. Electric and magnetic field power spectra of noise-cancelled peak segments demonstrate this, with values one to two orders of magnitude above the spectrum from a quiet segment (not shown).

After noise cancellation, the MT and magnetic transfer functions were estimated using the bounded influence remote reference algorithm of Chave \& Thomson (2004). This algorithm eliminates highly disturbed segments automatically provided there is minimal correlation between the corresponding electric (E) and magnetic (B) field components. Low correlation occurred when the B field was affected by tilt, therefore MT impedances (the ratio of E to B field) estimated from time-series after applying the noise-canceller showed generally small improvements over the estimates without application of the filter, with the only notable differences at the shortest periods $(<20 \mathrm{~s}$; not shown). Figure 11 shows the magnetic transfer responses (the ratio of vertical- to horizontal-component of the B field) for Lake Tete d'Ours estimated from the original and the cleaned time series, showing differences between both curves even at periods over $10 \mathrm{~s}$. In addition, the use of two stage processing with a clean remote reference site to remove correlated noise from the input (magnetic field) time series prior to estimation of the transfer functions (Chave and Thomson, 2004) was needed to further reduce the bias at the shortest periods, and for matching the curves obtained between the responses from the high and low sampling rate data in the period range of $100 \mathrm{~s}$ to $1000 \mathrm{~s}$. 


\section{TILT AND WIND CORRELATION: EFFECT OF WIND WAVES}

The Canadian Meteorological Service maintains four weather stations located in the western sector of the MT array where most of the sites from the first year survey are located. While the correlation scale of wind is typically a few meters, and hence a statistical correlation of the wind from a distant station with local tilt is not likely, correlation of intervals of high wind with large tilt variations does suggest a causative relation. Further, such a relation is suggested by the occurrence of disturbed tilt intervals during the warm months when the lakes are not frozen, and hence subject to the generation of wind waves.

We analyzed the summer and early fall months of August, 1998 and September, 1999, respectively, which are the times when the high sampling rate data from the two year-long surveys were recorded. During August 1998, the average wind direction at each weather station when the wind speed was over $15 \mathrm{~km} / \mathrm{h}$ is spatially homogeneous and points from the SSW, with mean values ranging from 23 to $27 \mathrm{~km} / \mathrm{h}$. In September 1999, the wind direction was more to the north (i.e., a southerly wind) with average wind speed very similar to that from August of the previous year.

Highly disturbed tilt events consistently coincide with high wind. This indicates that the wind is a driving force (direct or indirect) for water movements at the bottom of the lakes where the MT instruments are located. The highly disturbed segments with the largest tilt amplitude occurred when wind speeds were extremely high (usually with values exceeding $30 \mathrm{~km} / \mathrm{h}$ ), even for sites set beyond $20 \mathrm{~m}$ depth. Usually a time delay of a few hours is observed between peak wind and peak tilt. Peak winds (up to $55 \mathrm{~km} / \mathrm{h}$ ) typically last for about $3 \mathrm{~h}$, followed by a calmer interval of a few hours before high winds recur. However, tilt continues to be consistently disturbed despite the sequence of low and high wind speeds, usually lasting for up to two to three days (see data from Lake Tete d'Ours in Figure 12). Intuitively, instrument motion and/or local induction by wind-driven surface gravity waves are 
the most likely mechanisms for the observed tilt disturbance. Proving this would require data and models which are not readily available, notably wind data distributed around each lake, depth information about the lakes, and measurements of the electrical conductivity of lake water. It would also require a sophisticated model of the mechanical response of the lake bottom instruments to water motion over a range of spatial scales and frequencies. None of these are available, and hence recourse to simple bounds on the water motion produced by wind-induced waves and on the response of a cylindrical body to wave motion will be made.

Gravity wave response to wind forcing occurs over a continuum of frequencies, depending strongly on the fetch and water depth and on the wind growth. Wave prediction models are available for coastal areas which can be restricted in depth and laterally based on finite water depth linear theory (Automated Coastal Engineering System ACES 7B; http://www.veritechinc.com/navigate.htm), though these are only approximately applicable to the more tightly bounded geometry of the Slave lakes. Assuming wind speeds between 25 and $37 \mathrm{~km} / \mathrm{hr}$ lasting for three hours or longer (Fig.12), estimates of wave periods and wave amplitudes were obtained from this algorithm for combinations of input variables. The dominant variables were fetch (from 2 to $30 \mathrm{~km}$ ) and lake depth $(10$ to $50 \mathrm{~m}$ ), and of secondary importance the differential temperature between the air and water interface (a few degrees). Wave periods between 1-2 s and amplitudes of 0.2-0.4 $\mathrm{m}$ for the smallest lakes (fetch $<7 \mathrm{~km}$ ) and around 3-5 s period and 0.4-0.6 m amplitude for the largest Slave lakes (fetch of 10-20 km) were obtained.

Wave angular frequency $(\omega)$ and wavenumber $(k)$ for surface gravity waves are related through the finite water depth dispersion relation: $w^{2}=g k \tanh (k d)$, where $g$ is the acceleration of gravity and $d$ is the water depth. At a wave period of $4.5 \mathrm{~s}$ and a water depth of $20 \mathrm{~m}$, the wavenumber is 0.19887 , so that the wavelength is $31.6 \mathrm{~m}$. The deep water approximation is obtained when $k d>\pi$, so that $w^{2} \sim g k$, implying that deep water theory is appropriate for the lakes, assuming maximum wave periods 
of about $5 \mathrm{~s}$ if wind growth is the dominant force for surface gravity wave generation during high wind events. At a short wave period of $1 \mathrm{~s}$ (more likely at the smaller lakes) and water depth of $10 \mathrm{~m}$, the wavelength is $2 \mathrm{~m}$ and hence the deep water approximation becomes even more valid. At longer wave periods, deep water theory does not apply.

The deep water motion (orbital velocity $v$ ) from surface gravity waves decays exponentially with depth (z) according to $v=\omega A e^{-k z}$, where $A$ is the surface wave amplitude. Assuming a wave period of $3 \mathrm{~s}$ at an amplitude of $0.6 \mathrm{~m}$, the horizontal water particle displacement is $1.2 \times 10^{-8} \mathrm{~cm} / \mathrm{s}$ on a lake bed of $50 \mathrm{~m}$ depth (the deepest instrument deployment), $0.0074 \mathrm{~cm} / \mathrm{s}$ at $20 \mathrm{~m}$ depth (the general instrument depth), and $4 \mathrm{~cm} / \mathrm{s}$ at $6 \mathrm{~m}$ depth (the shallowest deployment). Hence surface gravity wave induced instrument motion may well account for the tilt oscillations.

Further insight can be obtained from the observation that the tilt motion is typically 3-4 times larger across the magnetometer cylinder (Y-axis) compared to along the cylinder (X-axis). This is consistent with the higher force needed to lift the larger cross-sectional area along $\mathrm{X}$ than is needed for lifting (or rotating in this case) along its cylinder diameter (Y). Karman vortex shedding of the magnetometer cylinder can be estimated from the nondimensional Strouhal number $S$, which is typically 0.22 in laminar flows (e.g., Newman, 1997). $S$ is the product of vortex frequency and cylinder diameter divided by water velocity. At a dominant period of $4.5 \mathrm{~s}$ and for a cylinder diameter of $23 \mathrm{~cm}$, the water velocity would have to be $23 \mathrm{~cm} / \mathrm{s}$ to produce $S \sim 0.22$. This is much higher than the velocities estimated for surface gravity waves, and also higher than any other likely disturbance. For example, internal waves for typical stratified lakes have estimated velocities between 1 and $10 \mathrm{~cm} / \mathrm{s}$ at the lake bed (Nepf, pers. comm., 2003). Unless the instrument is excited in one of the modes of its natural resonance frequency by the water mass passing by the cylinder, vortex shedding does not appear to be an explanation for the observed tilt.

Finally, we note that there are two sites which never displayed large summer-time 
tilt variations: Duncan and Kuuvik. The former is located close to Yellowknife where the wind speed was lower than at northern locations. Further, its fetch is negligible under the direction of strong wind measured at that time, suggesting that surface wave generation is weak. An explanation for the behavior of the very small Lake Kuuvik is lacking.

\section{MOTIONAL ELECTRIC FIELD FROM SURFACE GRAVITY WAVES}

We have shown in a previous section that some sites displayed electric as well as magnetic field variations correlated with tilt (Fig.10), hence suggesting electromagnetic induction by water motion.

The electric field amplitude induced by a moving medium (water in this case) is given by Ohm's law:

$$
\mathbf{E}^{\prime}=\mathbf{E}+\mathbf{v} \times \mathbf{B}
$$

(e.g., Larsen, 1971), where $\mathbf{E}^{\prime}$ is the electric field in a reference frame moving at velocity $\mathbf{v}, \mathbf{E}$ is the $\mathbf{E}$ field in a fixed reference frame, and $\mathbf{B}$ is the steady geomagnetic field. This provides the basic principle for estimation of motion-induced electric and magnetic fields within the ocean and lakes. The equation for the electric and magnetic fields induced by a plane progressive gravity wave in a flat-bottomed water body of uniform depth has been estimated by diverse authors (e.g., Weaver, 1965; Larsen, 1971). Assuming the thickness of the conductive water layer, sedimentary cover, and the lithosphere to be small compared to the wavelength of the incident field, and a negligible conductivity for the air and the lithosphere (underlain by a conductive mantle), Larsen (1971) derived the motional electric field induced by surface gravity waves. Assuming the Slave lakes may be approximated by a model with a uniform water depth and an infinite lateral extent like the ocean, the motional horizontal electric and vertical magnetic fields can be estimated by using the formula derived by 
Larsen (1971; Eqs.17 and 23). If we consider fresh water conductivity to range from 0.1 to $0.01 \Omega^{-1} \mathrm{~m}^{-1}$, subject to wind waves of period up to $5 \mathrm{~s}$ and wave amplitude up to $0.8 \mathrm{~m}$, with uniform water depth ranging from 7 to $10 \mathrm{~m}$, the horizontal electric field reaches a magnitude of around $0.2 \mathrm{mV} / \mathrm{km}$ and the vertical magnetic field amplitude is up to $0.01 \mathrm{nT}$. These estimates cannot explain the effect of electric field noise observed in the instruments deployed at $6 \mathrm{~m}$ depth in the large Aylmer Lake and at $10 \mathrm{~m}$ depth in the small Newbigging Lake, where the differences between a calm and a disturbed E field were on the order of $10-15 \mathrm{mV} / \mathrm{km}$ for Aylmer Lake and $\sim 8 \mathrm{mV} / \mathrm{km}$ for Newbigging. This suggests that the approach of a flat bottom for the Slave lakes is not appropriate and/or the water motion in these lake beds is not caused solely by surface gravity waves from wind driven forces. Considering wave periods beyond 5 s would cause the breakdown of Larsen's (or Weaver) equations, as these are based on intermediate and deep water wave theory, which are limited by the depth of the deployment $(10 \mathrm{~m})$ in this case. Considering E field amplitudes generated directly from the instrument motion, a simple calculation shows that the electrodes must move at improbably high speeds $(|\mathbf{v}|)$ of around $20 \mathrm{~cm} / \mathrm{s}$ for $\mathbf{v x B}$ (Eq.6) to be comparable to the E field disturbance observed. Such a speed is similar to the laminar flow velocity estimated from vortex shedding of the magnetometer. Is that a mere coincidence or is it because the instrument oscillated at some natural resonance frequency mode by the water motion? To answer this question will require data not available at present.

\section{SUMMARY AND DISCUSSION}

We have shown, from magnetotelluric (MT) surveys performed on the bottom of lakes of the Slave craton (NW Canada), that magnetic field data were disturbed by instrument motion that also registered in measurements of tilt. In some cases the electric field may also have been affected by electromagnetic induction generated by 
water motion.

The disturbances in the time series were highly correlated with tilt with a relatively consistent dominant period of around $4 \mathrm{~s}$, regardless of the depth and size of the lakes where the instruments were deployed. The magnetic and sometimes electric field channels can be pre-treated with an adaptive correlation cancellation filter using the two components of tilt as a reference signal. The method satisfactorily reduces the noise in the electromagnetic data under most circumstances. Exception are seen for some sites where the electric field noise was not negligible and hence there is correlated noise in the electric and magnetic channels. In this case, information on rotation of the instrument about the local vertical may be needed for an optimal noise cancellation. In addition, a two stage bounded influence processing algorithm with a clean remote reference site was generally required for processing of the filtered time series to obtain stable MT responses.

In order to reduce the instrument motion, it would be desirable to install the magnetometer in a solid package with a geometry that minimizes vortex shedding and thereby limits the oscillatory forces on it. In general, this means placing instruments close to the bottom with a minimum of buoyancy and the center of buoyancy located close to the center of mass of the anchor. In addition, a spherical case with small dimples like a golf ball can help reduce vorticity, although at the cost of increased drag force. Reduction of drag force upon the instrument can be achieved using a stiffer and heavier base (a rigid tripod of $\sim 25 \mathrm{~kg}$ was used in this survey). These considerations are of particular importance in coastal regions where sea bed currents are expected to be strong, for which tilt-induced electromagnetic fields from the water movement could be significant. 


\section{ACKNOWLEDGEMENT}

We would like to thank the Canadian Meteorological Service for providing the wind data, and Alan G Jones, Xavier Garcia, Jessica Spratt, Helmut Moeller, Jonathan Ware and John Bailey for their assistance in field work and in the data analysis. The advice given by Heidi Nepf (MIT) and Mark Grosenbaugh (WHOI) were valuable for gaining insight into the possible physical explanations for the instrument motion. Thanks also to Todd Gregory for useful suggestions to improve the manuscript. Commentaries given by the reviewers helped to improve the clarity of the manuscript, especially the review by L. Pedersen.

This project was funded by NSF grant EAR-9725556 and EAR-0087699. P.L. acknowledges the Fundacion Andes for a postdoctoral grant.

\section{REFERENCES}

Bastani, M., and L.B. Pedersen, The reliability of aeroplane attitude determination using the main geomagnetic field with application to tensor VLF data analysis, Geophysical Prospecting, 45, 831-841, 1997.

Chave, A.D., and D.J. Thomson, Bounded influence estimation of magnetotelluric response functions, Geophys. J. Int., 157, 988-1006, 2004.

Jones, A.G., P. Lezaeta, I.J. Ferguson, A.D. Chave b, R.L. Evans, X. Garcia \& J. Pratt, The electrical structure of the Slave craton, Lithos, 71, 505-527, 2003.

Larsen, J.C., The electromagnetic field of long and intermediate water waves, J. Mar. Res., 29, 28-45, 1971.

Newman, J.N., Marine Hydrodynamics, The MIT Press., Cambridge, Massachussets, pp.402, 1997. 
Petitt, R.A., Jr., Chave, A.D., Filloux, J.H., and Moeller, H.H., Electromagnetic field instrument for the continental shelf: Sea Technology, 35, 10-13, 1994.

Weaver, J.T., Magnetic variations associated with ocean waves and swell, J. Geophys. Res., 70, N.8, 1921-1929, 1965.

Widrow, B. and Stearns, S.D., Adaptive Signal Processing, Prentice-Hall, Inc., Englewoods Cliff, New Jersey, 1985. 


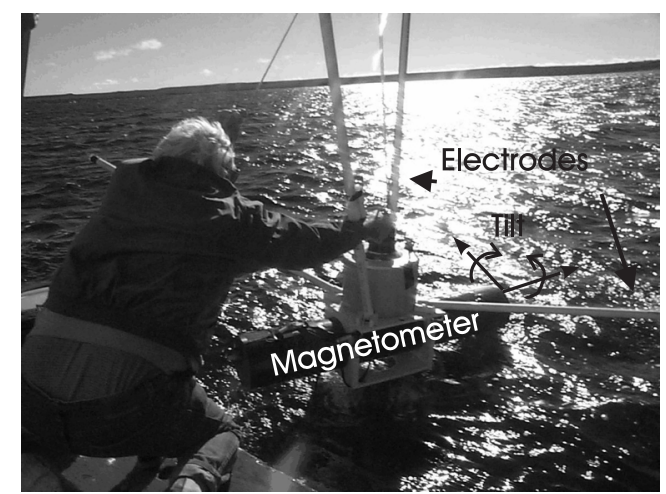

FIG. 1. A seafloor instrument used in the lake bottom surveys on the Slave craton being deployed from a twin Otter floatplane. Arrows indicate the parts of the instrument; the electrodes (at the ends of the two orthogonal light plastic pipes) are above the magnetometer and tilt sensor inside a horizontal cylindrical aluminum pressure case. Tilt is the angular deflection of the two horizontal principal coordinates of the cylinder, and are rotated by $45^{\circ}$ from the horizontal electrode pipes. The tripod anchor for the instrument is underwater. 


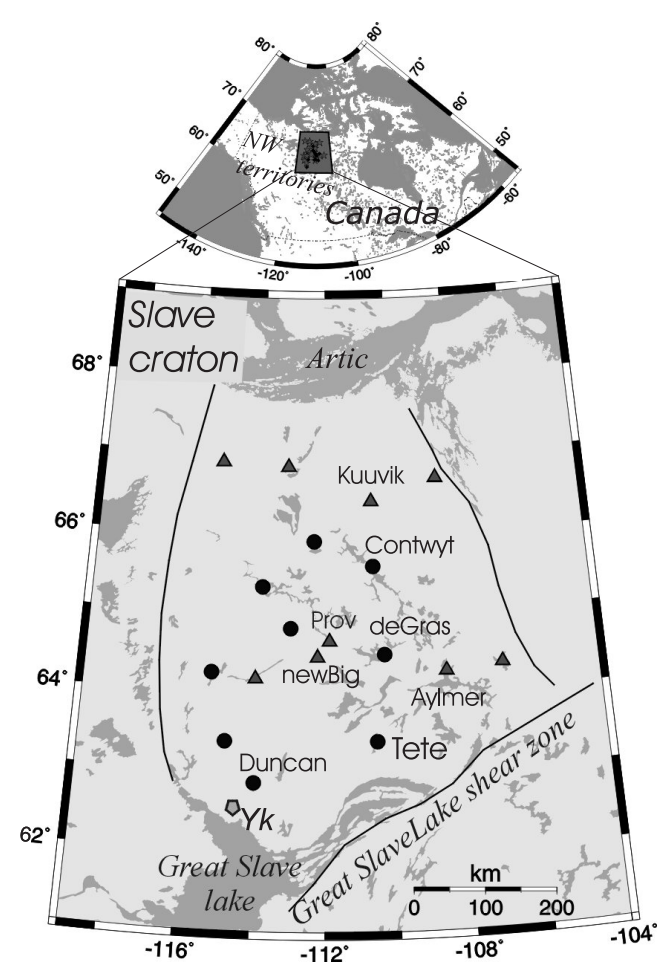

FIG. 2. Location of MT stations deployed on the bottom of lakes in the Slave craton (NW Canada). Black dots and triangles are sites from the first and second year surveys, respectively. YK: Weather station Yellowknife. Inset above shows a regional map of Canada. 


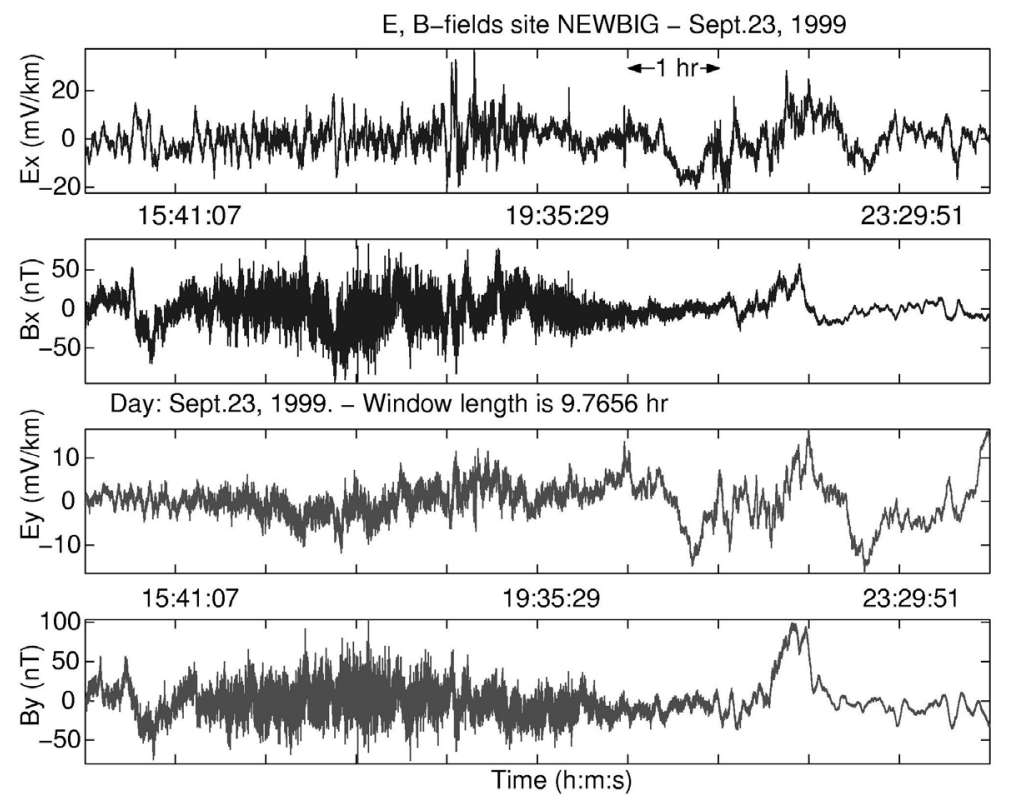

FIG. 3. Ten hour window of time series from Newbigging Lake (see Fig.2 for location) at a sample interval of $1.75 \mathrm{~s}$ for the 23rd of September'99. The two graphs at the top illustrate the original $\mathrm{X}$ component of the electric (Ex) and magnetic (Bx, along the magnetometer cylinder) channels. The two bottom graphs show the corresponding perpendicular Y component of the fields. E fields are at $45^{\circ}$ with respect to B. 

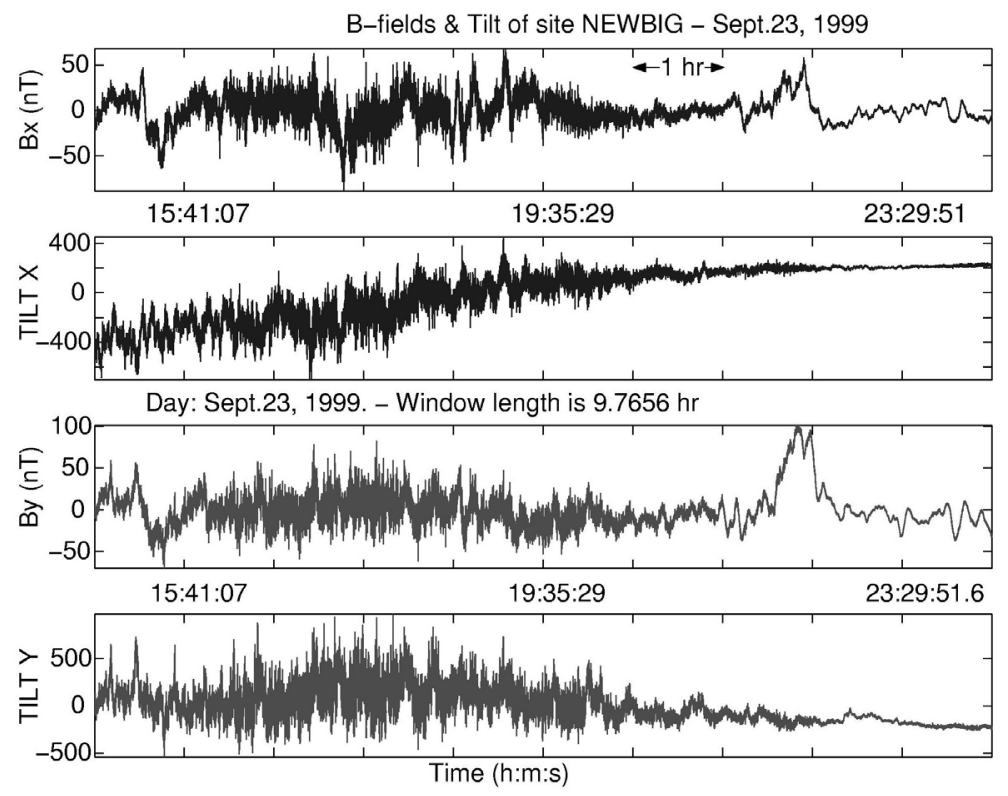

FIG. 4. Ten hour window of magnetic field and tilt variations from Newbigging Lake (see Fig.2 for location) at a sample interval of $1.75 \mathrm{~s}$ for the 23rd of September'99. The four traces from top to bottom illustrate the horizontal $\mathrm{X}$ component of the magnetic field $(\mathrm{Bx})$ and tilt (Tilt-x) and the Y component By and Tilt-y, respectively. Tilt is in microradians. The horizontal components are as for Figure 3. 


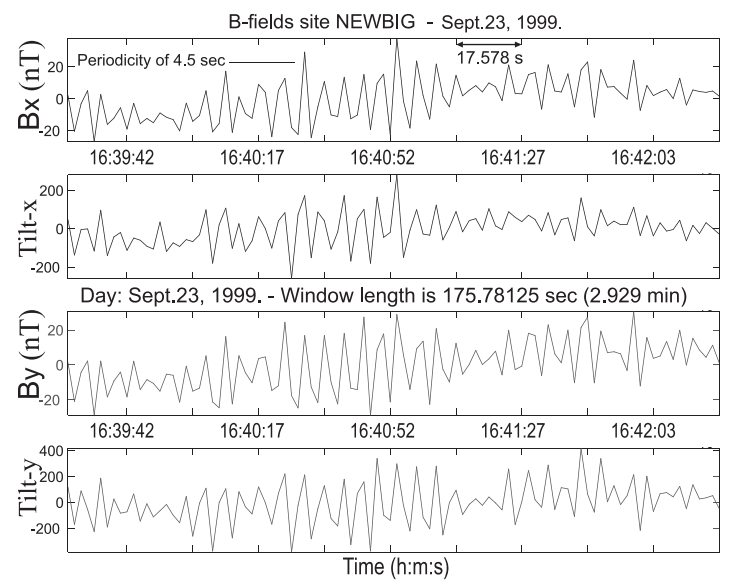

FIG. 5. Three minute window of magnetic field and tilt time variations from Newbigging Lake (see Fig.2 for location) at a sample interval of $1.75 \mathrm{~s}$ for the 23rd of September'99. The four traces from top to bottom illustrate the horizontal components as for Figure 4. 

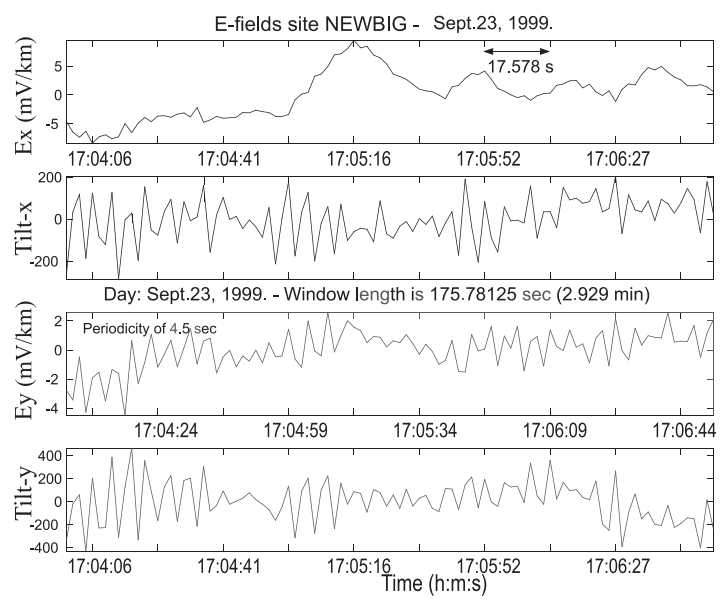

FIG. 6. Three minute window of the electric field (in the measurement coordinate system) and tilt time variations from Newbigging Lake (see Fig.2 for location) at a sample interval of $1.75 \mathrm{~s}$ for the 23rd of September'99. The four traces from top to bottom illustrate the horizontal components as for Figure 4. 

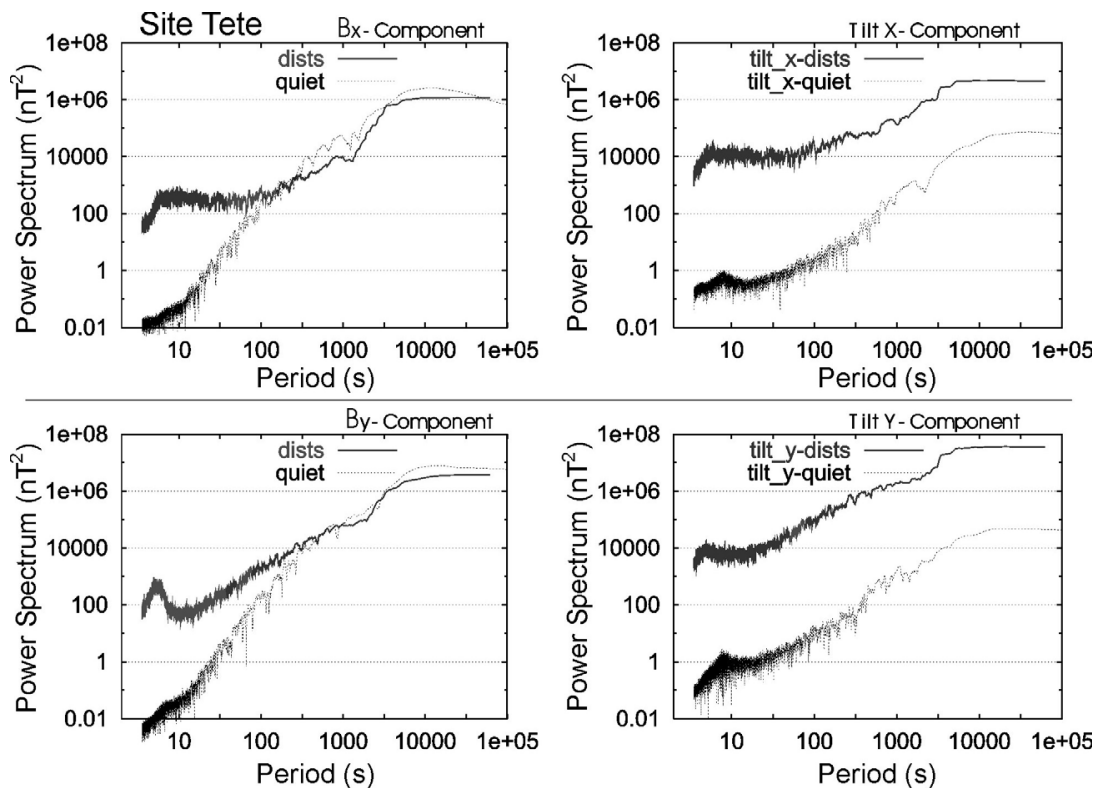

FIG. 7. Power spectra as a function of period for the magnetic fields components $B x$ and By (left) and the tilt components $X$ and $Y$ (right) for Lake Tete d'Ours. The two curves in each plot correspond to the power spectrum from highly disturbed (dists; 11-12 Aug'98) and relatively quiet tilt time segments (7-10 Aug'98). 


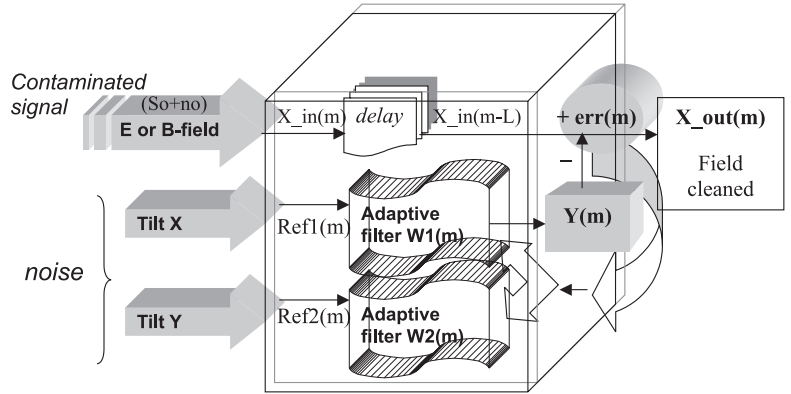

FIG. 8. Schematic showing a time domain adaptive filter to cancel tilt noise affecting the electric or magnetic field channels. err $(\mathrm{m})$ represents the residual uncorrelated signal which is the clean output signal (X_out) after convergence has been reached. 


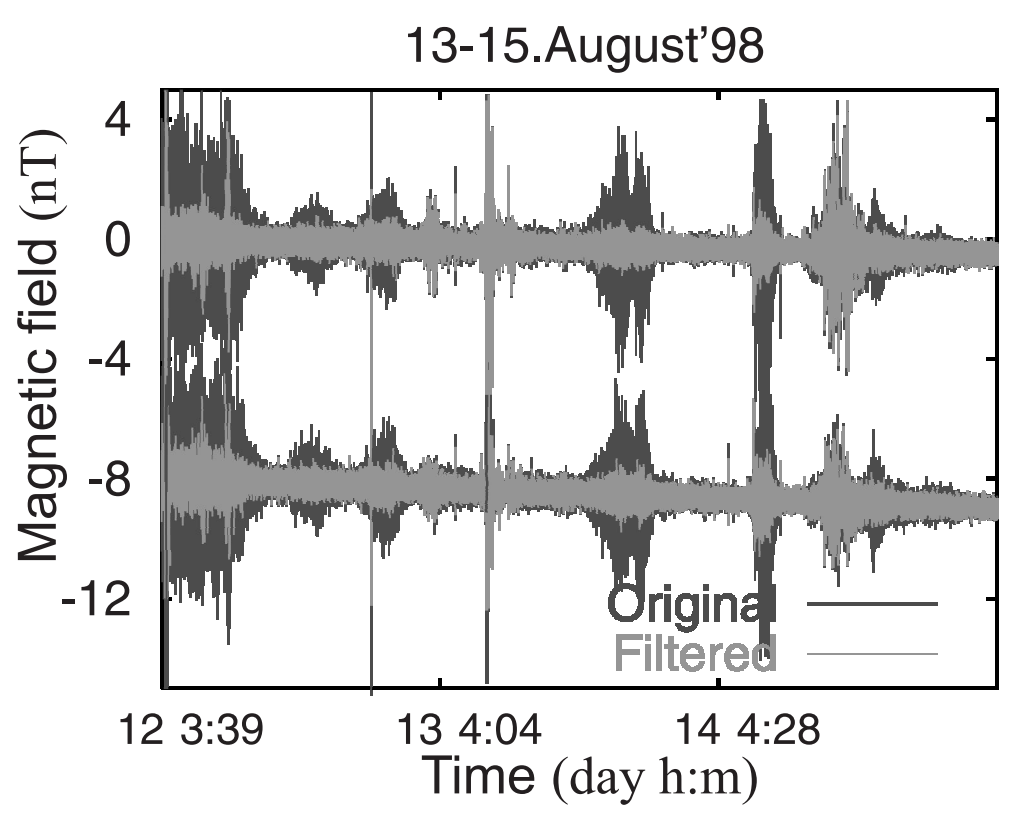

FIG. 9. Prewhitened time series of original (dark curve) and noise-cancelled horizontal components (in gray) for Lake Tete d'Ours in the measurement coordinate system. Curves from top and bottom are the magnetic Bx and By components at $106^{\circ}$ and $196^{\circ}$ from geographic north, respectively. The time window is during summer between the 13th and 15th of August, 1998. 

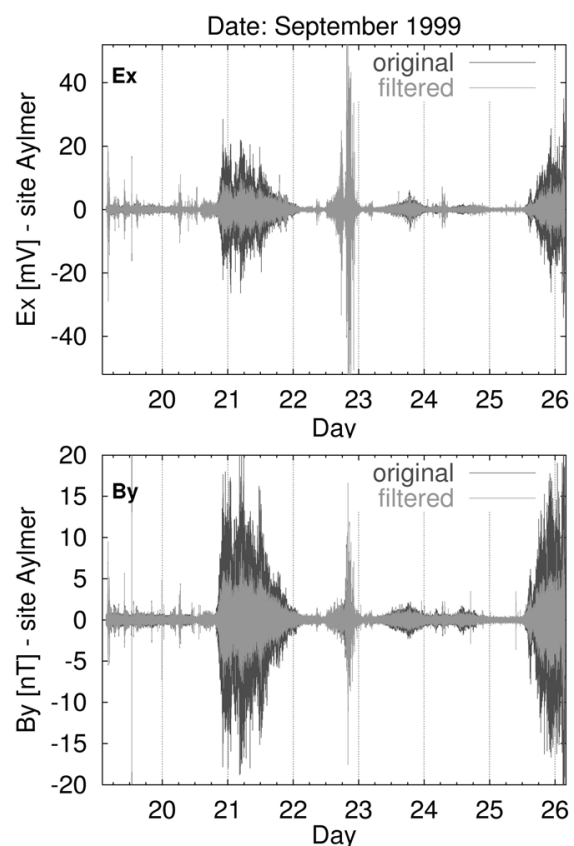

FIG. 10. Prewhitened time series of original (dark curve) and noise-cancelled horizontal components (in gray) for Aylmer Lake in the measurement coordinate system. Graphs from top and bottom are both the electric (Ex) and the magnetic (By) components at $185^{\circ}$ and $230^{\circ}$ from geographic north, respectively. The time window is during fall between the 19th and 26th of September, 1999. 


\section{Magnetic transfer functions from Lake Tete 'dours}
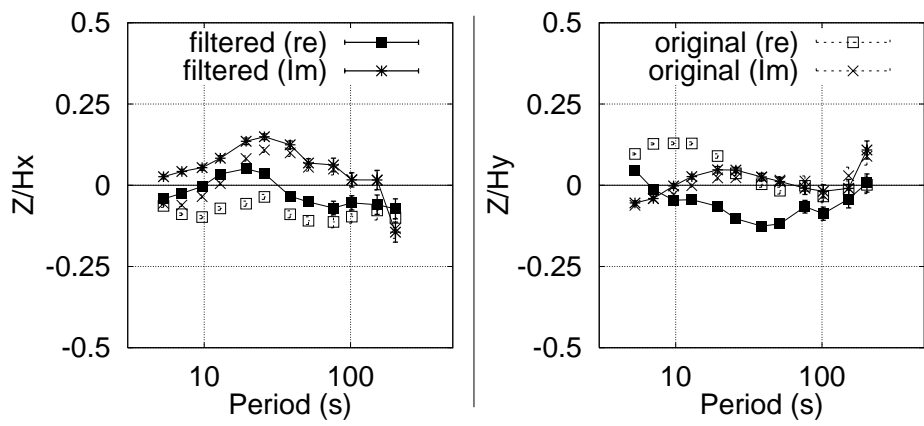

FIG. 11. Magnetic transfer functions for Lake Tete d'Ours estimated with a bounded influence algorithm (Chave \& Thomson, 2004). Symbols joined with lines are from cleaned (filtered) time series, while symbols alone are from the original time series without application of the noise canceler. Real parts are square symbols and imaginary parts are asterisks. 

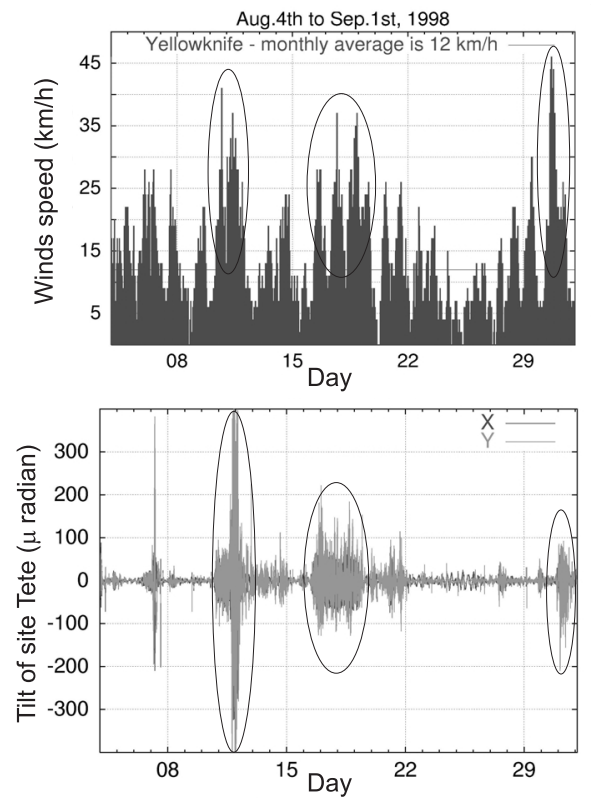

FIG. 12. Top: Hourly wind speeds at Yellowknife during August'98 and bottom the two components of prewhitened tilt (microradians) from Lake Tete d'Ours recorded during that same month. Ovals denote correlation between wind peaks and highly disturbed tilt. Location of the sites are shown in Figure 2. 